\title{
1 Gene discoveries in autism are biased towards comorbidity with intellectual
}

\section{2 disability}

3

4 Matthew Jensen ${ }^{1,2}$, Corrine Smolen ${ }^{1}$, and Santhosh Girirajan ${ }^{1,2,3}$

5 1. Department of Biochemistry and Molecular Biology, Pennsylvania State University, $6 \quad$ University Park, PA 16802, USA

7 2. Bioinformatics and Genomics Program, Huck Institute of the Life Sciences, Pennsylvania 8 State University, University Park, PA 16802, USA

9 3. Department of Anthropology, Pennsylvania State University, University Park, PA 16802, $10 \quad$ USA

\section{Correspondence to:}

14 Santhosh Girirajan

15 205A Life Sciences Building

16 Pennsylvania State University

17 University Park, PA 16802

18 E-mail: $\underline{\text { sxg47@psu.edu }}$

19 Phone: 814-865-0674 


\section{Abstract}

22 Autism typically presents with a highly heterogeneous set of features, including frequent

23 comorbidity with intellectual disability (ID). The overlap between these two phenotypes has

24 confounded the accurate diagnosis and discovery of genetic factors associated with autism. We

25 analyzed genetic variants in 2,290 individuals with autism from the Simons Simplex Collection

26 (SSC) who have either ID or normal cognitive function to determine whether genes associated

27 with autism also contribute towards ID comorbidity. We found that individuals who carried

28 variants in a set of 173 reported autism-associated genes showed decreased IQ $\left(\mathrm{p}=5.49 \times 10^{-6}\right)$ and

29 increased autism severity $(\mathrm{p}=0.013)$ compared with individuals without such variants. A subset

30 of autism-associated genes also showed strong evidence for ID comorbidity in published case

31 reports. We also found that individuals with high-functioning autism (IQ>100) had lower

32 frequencies of CNVs $(p=0.065)$ and LGD variants $(\mathrm{p}=0.021)$ compared with individuals who

33 manifested both autism and ID (IQ<70). These data indicated that de novo LGD variants

34 conferred a 1.53-fold higher risk $(\mathrm{p}=0.035)$ towards comorbid ID, while LGD mutations

35 specifically disrupting autism-associated genes conferred a 4.85 -fold increased risk $(\mathrm{p}=0.011)$ for

36 comorbid ID. Furthermore, de novo LGD variants in individuals with high-functioning autism

37 were more likely to disrupt genes with little functional relevance towards neurodevelopment, as

38 demonstrated by evidence from pathogenicity metrics, expression patterns in the developing

39 brain, and mouse model phenotypes. Overall, our data suggest that de novo pathogenic variants

40 disrupting genes associated with autism contribute towards autism and ID comorbidity, while

41 other genetic factors are likely to be causal for high-functioning autism. 
43 Autism spectrum disorder, which presents in children with social communication difficulties,

44 repetitive behavior, and restricted interests ${ }^{1}$, is a highly heterogeneous neurodevelopmental

45 disorder characterized by complex genetic etiology and strong comorbidity with other

46 developmental disorders ${ }^{2}$. For example, approximately $30 \%$ of individuals with autism also

47 manifest with intellectual disability (ID) ${ }^{3}$, defined ${ }^{1}$ by IQ scores $<70$. The high degree of co-

48 occurrence of autism with ID has been shown to confound accurate diagnosis of autism. In fact,

49 we recently showed that $69 \%$ of individuals diagnosed with ID are likely to be recategorized and

50 diagnosed with autism ${ }^{4}$. The diagnostic overlap between autism and ID suggests that de novo

51 gene disruptive variants and copy-number variants $(\mathrm{CNVs})$ identified in individuals ascertained

52 for autism in large-scale studies could also be confounded by ID comorbidity. Here, using

53 genetic and phenotypic data from 2,290 individuals with autism from the Simons Simplex

54 Collection $(\mathrm{SSC})^{5}$, we show that gene discoveries in autism are biased towards genes that

55 contribute towards both autism and comorbid ID.

56 We analyzed rare de novo likely-gene disruptive (LGD) variants from exome sequencing

57 data $^{6,7}$, disease-associated copy-number variants (CNVs) from microarrays ${ }^{8}$, and Full-scale IQ

58 and Social Responsiveness Scale (SRS) T-scores for SSC probands that were obtained from the

59 Simons Foundation Autism Research Initiative ${ }^{5}$. As these data were de-identified, they were

60 exempt from IRB review and conformed to the Helsinki Declaration. We first compared the

61 phenotypes of 288 individuals with de novo LGD variants and 81 individuals with pathogenic

$62 \mathrm{CNVs}$ to 1,921 individuals without such variants obtained from the SSC cohort. Similar to

63 previous autism studies that identified correlations between de novo variants and IQ scores ${ }^{9-12}$,

64 we found that individuals with de novo LGD variants (IQ=77.7, $\mathrm{p}=0.031$, two-tailed Mann-

65 Whitney test) or pathogenic $\mathrm{CNVs}(\mathrm{IQ}=76.3, \mathrm{p}=0.002)$ had a significant decrease in IQ scores

66 compared with individuals without such variants $(\mathrm{IQ}=82.3)$ (Figure 1A). However, no

67 differences in autism severity, measured using SRS T-scores, were observed between groups of

68 individuals with and without pathogenic variants $(p=0.104$ for LGD variants and 0.963 for

$69 \mathrm{CNVs}$ ) (Figure 1A). This suggests that pathogenic variants in general contribute to ID

70 independent of autism severity, although this could also be due to an ascertainment bias in the

71 SSC cohort towards individuals with severe autism.

72 We further identified individuals carrying de novo LGD variants in 173 autism-associated

73 genes, defined as genes with recurrent de novo variants reported in multiple databases of 
74 sequencing studies (Table S1). These genes included tier 1 genes ( $>2$ de novo LGD variants)

75 from the Developing Brain Disorders Gene Database ${ }^{13}$, genes with $>5$ non-SSC de novo LGD

76 variants from denovo-db ${ }^{14}$, and SFARI Gene tiers 1 and 2 (https://gene.sfari.org/). We found that

77 individuals carrying de novo LGD variants in autism-associated genes had decreased IQ ( $\mathrm{n}=74$,

$78 \mathrm{IQ}=69.1, \mathrm{p}=5.49 \times 10^{-6}$, two-tailed Mann-Whitney test) and increased SRS T-scores (SRS=82.4,

$79 \mathrm{p}=0.013)$ compared with individuals without $L G D$ variants $(\mathrm{n}=2,216, \mathrm{IQ}=81.9, \mathrm{SRS}=79.6)$,

80 implying that candidate autism genes contribute to both autism and ID phenotypes (Figure 1B).

81 To validate this finding, we examined 76 published case reports of affected individuals with

82 pathogenic variants in a subset of 22 autism genes that appeared in all three autism gene

83 databases (Table 1, Table S2). For example, recent case studies have identified autism co-

84 occurring with ID in 21 individuals with de novo SHANK3 variants ${ }^{15}$, 19 individuals with

$85 N R X N 1$ variants $^{16}$, and 18 individuals with TCF20 variants ${ }^{17}$. Overall, 460/497 (92.6\%)

86 individuals with autism described in these studies had ID features, emphasizing that variants in

87 these genes contribute to a severe form of autism with comorbid ID (Table 1).

We next compared genetic data from 397 SSC individuals (17.3\% of the SSC cohort) with "high-functioning autism", defined as having severe autism and average or above-average IQ scores (SRS>75 and IQ>100), to 562 individuals (24.5\%) with both autism and ID (SRS>75 and IQ<70). Individuals with high-functioning autism had a significantly lower $(\mathrm{p}=0.021$, onetailed Fisher's Exact test) frequency of de novo LGD variants (42/397, 10.6\%) than individuals with autism and ID (86/562, 15.3\%). Similarly, individuals with high-functioning autism were less likely $(\mathrm{p}=0.065)$ to carry pathogenic CNVs $(9 / 397,2.3 \%)$ than individuals with autism and ID (24/562, 4.3\%). In fact, de novo LGD variants conferred a 1.53-fold higher likelihood of manifesting ID among individuals with autism ( $\mathrm{p}=0.035,95 \%$ confidence interval 1.03-2.26), and pathogenic CNVs similarly conferred a 1.92-fold increased risk for co-occurrence of ID among individuals with autism ( $\mathrm{p}=0.099,95 \%$ CI 0.88-4.18). We replicated these observations by analyzing an additional combined cohort of 2,357 individuals from both the SSC and the Autism Sequencing Collection ${ }^{18}$. Here, individuals with autism and ID had a significantly higher rate ( $\mathrm{p}=3.04 \times 10^{-6}$, one-tailed Student's t-test) of de novo variants in genes intolerant to variation,

103 individuals manifesting autism but not ID (114/1747, 6.65\%). We also found that only $3 / 397$

$104(0.8 \%)$ individuals in the SSC cohort with high-functioning autism carried de novo LGD variants 
105 in autism-associated genes, including $A N K 2, H I V E P 3$, and $B A Z 2 B$. This frequency was not

106 significantly different from the expected frequency of de novo variants in the general population

107 ( $\mathrm{p}=0.095$, one-tailed Student's T-test), as calculated from gene-specific probabilities of de novo

108 nonsense and frameshift variants from a sequence context-dependent $\operatorname{model}^{9}$. In contrast, 20/562

109 (3.6\%) individuals with autism comorbid with ID carried de novo LGD variants in autism-

110 associated genes, such as $C H D 8, S C N 2 A$, and $S Y N G A P 1$, representing a 19.2-fold enrichment of

111 variants compared with the expected rate in the general population $\left(\mathrm{p}=9.48 \times 10^{-6}\right)$. Thus, de novo

112 LGD variants in autism genes conferred a 4.85-fold increased risk ( $\mathrm{p}=0.011,95 \%$ CI 1.43-16.42)

113 towards comorbid ID in individuals with autism.

114 We further sought to determine the biological relevance of the 42 genes with de novo

115 LGD variants identified in individuals with high-functioning autism, and found that these genes

116 in aggregate had less functional relevance towards neurodevelopment than the reported autism-

117 associated genes. For example, genes with de novo LGD variants in individuals with high-

118 functioning autism were less resistant to genetic variation than reported autism-associated genes,

119 as measured by Residual Variation Intolerance Score (RVIS) ( $p=4.00 \times 10^{-4}$, Mann-Whitney two-

120 tailed test) and $p L I$ percentile $\left(p=9.77 \times 10^{-7}\right)$ gene metrics ${ }^{19,20}$ (Figure 2A). In fact, while the

121 RVIS and pLI percentiles of the reported autism genes were clustered below the thresholds for

122 pathogenicity (RVIS $<20^{\text {th }}$ percentile and $\mathrm{pLI}<18^{\text {th }}$ percentile, or raw score $>0.9$ ), genes

123 disrupted among individuals with high-functioning autism were evenly distributed across the

124 range of percentiles. Additionally, we tested the enrichment of each gene set for specific

125 expression in brain regions during development, based on expression data derived from the

126 BrainSpan Atlas ${ }^{21}$, using the Specific-Expression Analysis (SEA) online tool ${ }^{22}$. While autism

127 genes were enriched for specific expression in the cortex $\left(p=3.13 \times 10^{-4}\right.$, Fisher's Exact test with

128 Benjamini-Hochsberg correction) and cerebellum $(\mathrm{p}=0.020)$ during early fetal development ${ }^{22}$,

129 genes with de novo LGD variants in high-functioning autism individuals were not enriched for

130 any specific expression patterns in the developing brain (Figure 2B). Furthermore, mouse

131 models of genes identified in individuals with high-functioning autism, whose phenotypic data

132 were obtained from the Mouse Genome Informatics database ${ }^{24}$, were significantly less likely to

133 manifest nervous system $\left(p=4.90 \times 10^{-3}\right.$, one-tailed Fisher's Exact test with Benjamini-Hochsberg

134 correction) and behavioral/neurological $(\mathrm{p}=0.037)$ phenotypes than mouse models of reported

135 autism-associated genes (Figure 2C). These findings suggest that genes with de novo LGD 
variants in individuals with high-functioning autism are less pathogenic in humans and model organisms, and therefore may not necessarily contribute towards the specific high-functioning autism phenotype.

Our data indicate that pathogenic variants such as de novo LGD variants and CNVs contribute to autism phenotypes primarily in individuals with comorbid ID, especially if the variants disrupt a gene previously associated with autism. Several themes regarding the study of

142 high-functioning autism have emerged from these findings. First, the consistently high degree of 143 comorbidity between autism and ID has led to an ascertainment bias towards individuals who 144 manifest both disorders in large-scale sequencing cohorts, as it is difficult to exclude all 145 individuals with comorbid disorders and still have adequate power to identify recurrent variants. 146 Indeed, more than $80 \%$ of the SSC cohort had an IQ score less than 100, and the average IQ of 147 the cohort (81.5) was 18.5 points below the population average. This bias has contributed to the 148 identification of genes and CNV regions related to both autism and ID, as evidenced by the 149 decreased IQ among carriers of variants in these genes as well as a high incidence of comorbid 150 phenotypes reported in published case studies. Large-scale sequencing studies still hold a high 151 value in uncovering shared biological mechanisms that could underlie both disorders ${ }^{23}$. 152 However, understanding the biology of the core autism phenotypes would require concerted 153 efforts to recruit individuals who specifically manifest high-functioning autism without ID. Second, individuals with high-functioning autism are less likely to carry de novo LGD variants in candidate autism genes, and de novo variants in individuals with high-functioning 156 autism tend to disrupt genes with less functional relevance towards neurodevelopment. These 157 genes likely carry non-recurrent variants that either confer a small effect size towards autism risk 158 on their own, or are not associated at all with neurodevelopment. We therefore propose that 159 multiple genomic factors with varying effect sizes, such as missense variants, common variants, 160 variants in regulatory and non-coding regions, or the combinatorial effects of inherited variants, 161 contribute towards autism phenotypes without ID. For example, Schaaf and colleagues 162 performed targeted sequencing of 21 candidate autism genes in 339 individuals with high163 functioning autism ${ }^{25}$. They found that $2 \%$ of individuals carried de novo missense variants in 164 candidate autism genes, such as PTEN and FOXP2, suggesting that allelic variants of differing 165 severity within the same gene might contribute to distinct neurodevelopmental trajectories. 166 Interestingly, the same study also found that $7 \%$ of individuals with high-functioning autism 
167 carried multiple inherited missense variants in candidate autism genes, potentially contributing to

168 an oligogenic model for high-functioning autism phenotypes. Similarly, common variants have

169 been found to contribute towards increased autism risk in individuals without $\mathrm{ID}^{26,27}$. For

170 example, Grove and colleagues recently reported that the heritability attributed to common

171 variants, including those primarily associated with cognitive ability and educational attainment,

172 was three times lower in individuals with autism and ID compared with those without ID $^{27}$.

173 Finally, variants that may not contribute directly towards autism phenotypes themselves,

174 including the de novo LGD variants observed in individuals with high-functioning autism, could 175 still be responsible for subtler modification of the severity of autism or ID phenotypes.

176 Overall, our results emphasize the importance of dissecting phenotypic heterogeneity in

177 family-based sequencing studies of complex diseases, especially those with a high frequency of

178 comorbid disorders. While a larger cohort of individuals recruited specifically for high-

179 functioning autism could identify associations with recurrent genes or different types of variants,

180 these findings should be validated using functional studies to more fully differentiate the genetic

181 causes for high-functioning autism from those for autism with comorbid ID. 


\section{Supplemental data}

184 Supplemental data include two supplemental tables in Excel file format.

\section{Declaration of Interests}

187 The authors declare that they have no conflicts of interest.

\section{Acknowledgements}

190 This work was supported by NIH R01-GM121907, SFARI Pilot Grant (\#399894) and resources

191 from the Huck Institutes of the Life Sciences to S.G., and NIH T32-GM102057 to M.J. The

192 authors thank Fereydoun Hormozdiari (UC Davis), Lucilla Pizzo (Penn State), and Vijay Kumar

193 (Penn State) for their helpful discussions and comments on the manuscript. We are grateful to all

194 of the families at the participating Simons Simplex Collection (SSC) sites, as well as the

195 principal investigators (A. Beaudet, R. Bernier, J. Constantino, E. Cook, E. Fombonne, D.

196 Geschwind, R. Goin-Kochel, E. Hanson, D. Grice, A. Klin, D. Ledbetter, C. Lord, C. Martin, D.

197 Martin, R. Maxim, J. Miles, O. Ousley, K. Pelphrey, B. Peterson, J. Piggot, C. Saulnier, M.

198 State, W. Stone, J. Sutcliffe, C. Walsh, Z. Warren, E. Wijsman). We appreciate obtaining access

199 to phenotypic data on the Simons Foundation Autism Research Initiative (SFARI) Base.

200 Approved researchers can obtain the SSC data sets described in this study by applying at

201 https://www.base.sfari.org.

202

\section{Author Contributions}

204 M.J. and S.G. conceptualized the study, and M.J. and C.S. analyzed the data. M.J. and S.G. wrote 205 the manuscript with input from all authors. 


\section{References}

1. American Psychiatric Association (2013). Diagnostic and Statistical Manual of Mental

Disorders (American Psychiatric Association).

2. Vorstman, J.A.S., Parr, J.R., Moreno-De-Luca, D., Anney, R.J.L., Nurnberger Jr, J.I., and Hallmayer, J.F. (2017). Autism genetics: opportunities and challenges for clinical translation.

214 Nat. Rev. Genet. 18, 362-376.

3. Baio, J., Wiggins, L., Christensen, D.L., Maenner, M.J., Daniels, J., Warren, Z., Kurzius-

217 Spencer, M., Zahorodny, W., Robinson, C., Rosenberg, et al. (2018). Prevalence of Autism 218 Spectrum Disorder Among Children Aged 8 Years - Autism and Developmental Disabilities

219 Monitoring Network, 11 Sites, United States, 2014. MMWR. Surveill. Summ. 67, 1-23. confounds ascertainment of autism: implications for genetic diagnosis. Am. J. Med. Genet. Part

223 B Neuropsychiatr. Genet. 168, 600-608. Identification of Autism Genetic Risk Factors. Neuron 68, 192-195.

229 Witherspoon, K.T., Vives, L., Patterson, K.E., et al. (2014). The contribution of de novo coding 230 mutations to autism spectrum disorder. Nature 515, 216-221.

233 B.P., Stessman, H.A., He, Z.X., et al. (2015). Excess of rare, inherited truncating mutations in 234 autism. Nat. Genet. 47, 582-588.

236 8. Sanders, S.J., He, X., Willsey, A.J., Ercan-Sencicek, A.G., Samocha, K.E., Cicek, A.E., 237 Murtha, M.T., Bal, V.H., Bishop, S.L., Dong, S., et al. (2015). Insights into Autism Spectrum 
Disorder Genomic Architecture and Biology from 71 Risk Loci. Neuron 87, 1215-1233.

9. Samocha, K.E., Robinson, E.B., Sanders, S.J., Stevens, C., Sabo, A., McGrath, L.M., interpretation of de novo mutation in human disease. Nat. Genet. 46, 944-950. Daly, M.J. (2014). Autism spectrum disorder severity reflects the average contribution of de novo and familial influences. Proc. Natl. Acad. Sci. 111, 15161-15165. Whitney, J., Deflaux, N., Bingham, J., Wang, Z., et al. (2017). Whole genome sequencing resource identifies 18 new candidate genes for autism spectrum disorder. Nat. Neurosci. 20, 602611. modulate cognitive and developmental phenotypes in individuals carrying disease-associated variants. Genet. Med. 21, 816-825. cross-disorder method to identify novel candidate genes for developmental brain disorders. JAMA Psychiatry 73, 275-283.

264 human de novo variants. Nucleic Acids Res. 45, D804-D811.

267 (2018). Genotype and phenotype correlations for SHANK3 de novo mutations in 
16. Al Shehhi, M., Forman, E.B., Fitzgerald, J.E., McInerney, V., Krawczyk, J., Shen, S., Betts,

272 phenotypic and penetrance data from 34 families. Eur. J. Med. Genet. 62, 204-209.

273

274 17. Torti, E., Keren, B., Palmer, E.E., Zhu, Z., Afenjar, A., Anderson, I.J., Andrews, M. V.,

275 Atkinson, C., Au, M., Berry, S.A., et al. (2019). Variants in TCF20 in neurodevelopmental

276 disability: description of 27 new patients and review of literature. Genet. Med. ePub ahead of

277 print.

18. Kosmicki, J.A., Samocha, K.E., Howrigan, D.P., Sanders, S.J., Slowikowski, K., Lek, M., samples. Nat. Genet. 49, 504-510.

20. Lek, M., Karczewski, K.J., Minikel, E. V., Samocha, K.E., Banks, E., Fennell, T.,

289 O’Donnell-Luria, A.H., Ware, J.S., Hill, A.J., Cummings, B.B., et al. (2016). Analysis of 
300 23. Jensen, M., and Girirajan, S. (2017). Mapping a shared genetic basis for neurodevelopmental 301 disorders. Genome Med. 9, 109.

302

303 24. Smith, C.L., Blake, J.A., Kadin, J.A., Richardson, J.E., Bult, C.J., and Mouse Genome

304 Database Group (2018). Mouse Genome Database (MGD)-2018: knowledgebase for the

305 laboratory mouse. Nucleic Acids Res. 46, D836-D842.

307 25. Schaaf, C.P., Sabo, A., Sakai, Y., Crosby, J., Muzny, D., Hawes, A., Lewis, L., Akbar, H., 308 Varghese, R., Boerwinkle, E., et al. (2011). Oligogenic heterozygosity in individuals with high309 functioning autism spectrum disorders. Hum. Mol. Genet. 20, 3366-3375.

311 26. Weiner, D.J., Wigdor, E.M., Ripke, S., Walters, R.K., Kosmicki, J.A., Grove, J., Samocha,

312 K.E., Goldstein, J.I., Okbay, A., Bybjerg-Grauholm, J., et al. (2017). Polygenic transmission

313 disequilibrium confirms that common and rare variation act additively to create risk for autism

314 spectrum disorders. Nat. Genet. 49, 978-985.

316 27. Grove, J., Ripke, S., Als, T.D., Mattheisen, M., Walters, R.K., Won, H., Pallesen, J., Agerbo, 317 E., Andreassen, O.A., Anney, R., et al. (2019). Identification of common genetic risk variants for 318 autism spectrum disorder. Nat. Genet. 51, 431-444. 


\section{Figure Legends}

321 Figure 1. Phenotypic comparison of individuals with autism from the SSC cohort with and

322 without pathogenic variants. (A) Individuals with pathogenic variants (de novo LGD and CNV)

323 had a significantly lower IQ than individuals without pathogenic variants, but no change in

324 autism severity (SRS T-score) was observed between the three groups. (B) Individuals with de

325 novo LGD variants in candidate autism genes had a lower IQ and more severe autism phenotypes

326 than individuals without such variants. $\mathrm{n}$ indicates sample size, $\mathrm{p}$-values were derived from two-

327 tailed Mann-Whitney tests, and dotted lines within each plot indicate the median and first and

328 third quartiles. All statistics were calculated using R v.3.4.2 (R Foundation for Statistical

329 Computing, Vienna, Austria).

331 Figure 2. Functional analysis of genes with de novo LGD variants in individuals with high-

332 functioning autism. (A) Genes with de novo LGD variants in individuals with high-functioning 333 autism had lower average RVIS (left) and pLI (right) percentile scores than those for reported 334 autism-associated genes. Thick dotted lines across the violin plots indicate thresholds for gene 335 pathogenicity: $<20^{\text {th }}$ percentile for RVIS and $<18^{\text {th }}$ percentile for $p L I$ ( $>0.9$ raw score). Thin lines

336 within the violin plots indicate the median and first and third quartiles. p-values were derived

337 from two-tailed Mann-Whitney tests. (B) Expression of genes with de novo variants in

338 individuals with high-functioning autism and autism-associated genes in the developing human

339 brain. Autism-associated genes were enriched for specific expression in the cortex and

340 cerebellum during early development, while no enrichment was seen in the genes identified in

341 individuals with high-function autism. Hexagon sizes represent the number of genes

342 preferentially expressed in each brain tissue and timepoint, while colors of the hexagons

343 represents p-values for the enrichment of autism genes among each set of preferentially-

344 expressed genes. (C) Frequency of phenotypes observed in mouse knockout models for genes

345 with de novo LGD variants in individuals with high-functioning autism compared with reported

346 autism-associated genes. * indicates $\mathrm{p}<0.05$ with Benjamini-Hochsberg correction. 
348 Table 1. Individuals carrying variants in autism-associated genes with comorbid ID.

\begin{tabular}{|c|c|c|c|}
\hline $\begin{array}{l}\text { Autism- } \\
\text { associated genes }\end{array}$ & Cases with ID & $\begin{array}{c}\text { Autism cases } \\
\text { with or without } \\
\text { ID }\end{array}$ & $\begin{array}{c}\text { Autism cases } \\
\text { with comorbid } \\
\text { ID }\end{array}$ \\
\hline$A D N P$ & $134 / 134$ & $114 / 134$ & $114 / 114$ \\
\hline$A N K 2$ & $1 / 1$ & $1 / 1$ & $1 / 1$ \\
\hline ANKRD11 & $10 / 10$ & $9 / 10$ & $9 / 9$ \\
\hline$A R I D 1 B$ & $137 / 153$ & $80 / 153$ & $80 / 80$ \\
\hline$A S H 1 L$ & $14 / 14$ & $4 / 14$ & $4 / 4$ \\
\hline$A S X L 3$ & $18 / 19$ & $16 / 19$ & $15 / 16$ \\
\hline$B C L 11 A$ & $11 / 16$ & $4 / 16$ & $3 / 4$ \\
\hline CHD2 & $3 / 3$ & $3 / 3$ & $3 / 3$ \\
\hline CHD 8 & $56 / 75$ & $61 / 75$ & $46 / 61$ \\
\hline CUL3 & $1 / 1$ & $1 / 1$ & $1 / 1$ \\
\hline$D D X 3 X$ & $97 / 97$ & $33 / 97$ & $33 / 33$ \\
\hline DYRK1A & $7 / 26$ & $9 / 26$ & $7 / 9$ \\
\hline$K M T 2 A$ & 76/99 & $12 / 99$ & $10 / 12$ \\
\hline$M E C P 2$ & $1 / 1$ & $1 / 1$ & $1 / 1$ \\
\hline MYT1L & $1 / 1$ & $1 / 1$ & $1 / 1$ \\
\hline$N R X N 1$ & $45 / 60$ & $34 / 60$ & $23 / 34$ \\
\hline POGZ & $48 / 49$ & $29 / 49$ & $29 / 29$ \\
\hline$S C N 2 A$ & $19 / 33$ & $9 / 33$ & $7 / 9$ \\
\hline SETD5 & $15 / 16$ & $7 / 16$ & $7 / 7$ \\
\hline SHANK3 & $35 / 37$ & $26 / 37$ & $24 / 26$ \\
\hline SYNGAP1 & $11 / 23$ & $11 / 23$ & $11 / 11$ \\
\hline TCF20 & $47 / 48$ & $32 / 48$ & $31 / 32$ \\
\hline Total & $787 / 916(85.9 \%)$ & $497 / 916(54.3 \%)$ & $460 / 497(92.6 \%)$ \\
\hline
\end{tabular}




\section{Figure 1}

A

Phenotypic severity of individuals with autism carrying pathogenic variants

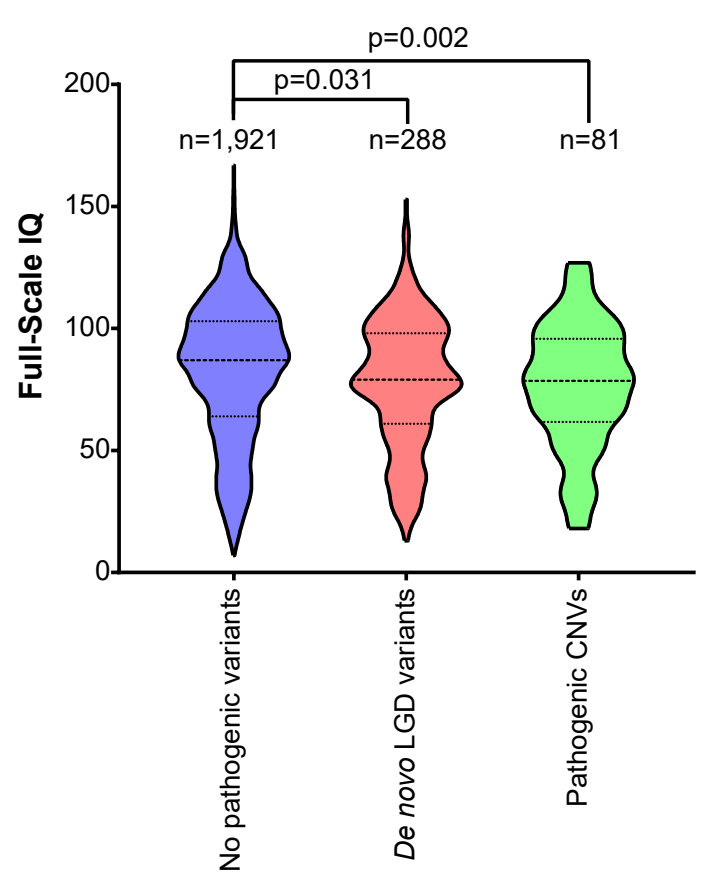

B

Phenotypic severity of individuals carrying variants in autism genes
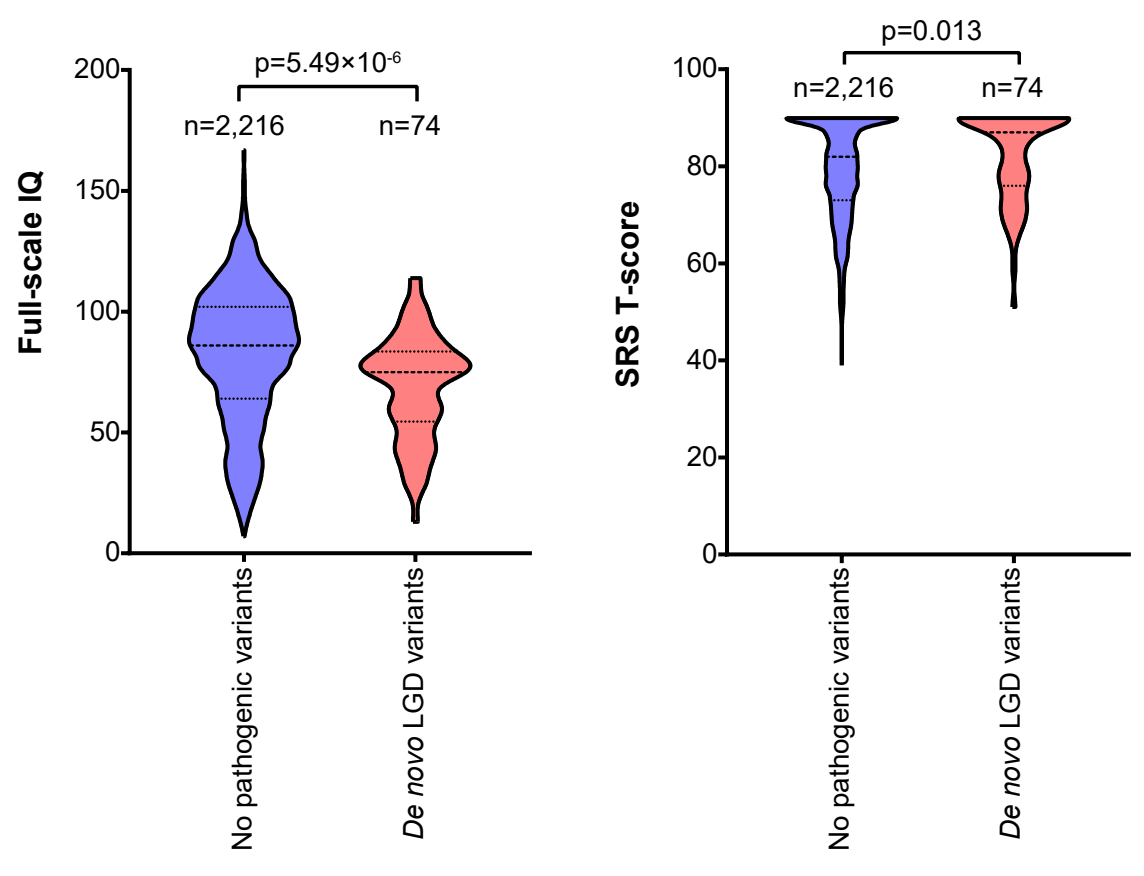


\section{Figure 2}

A

Pathogenicity metrics of autism genes

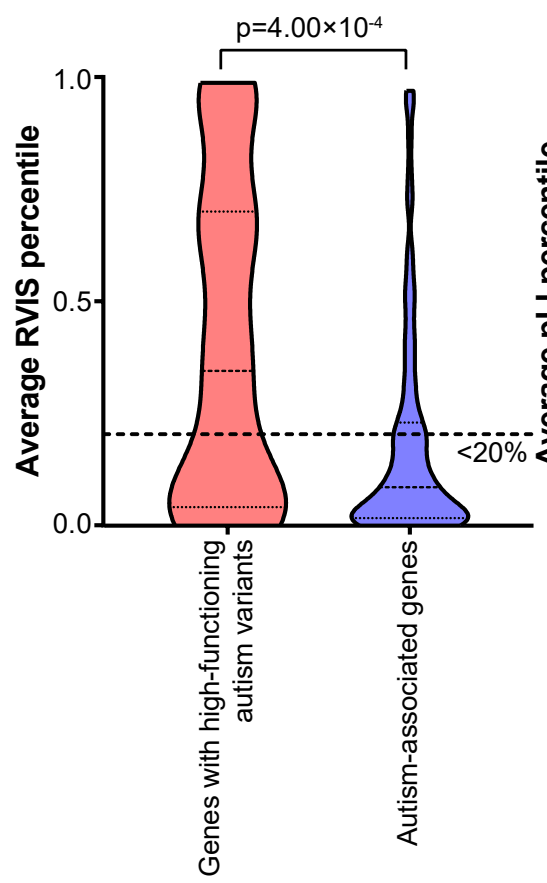

C
B

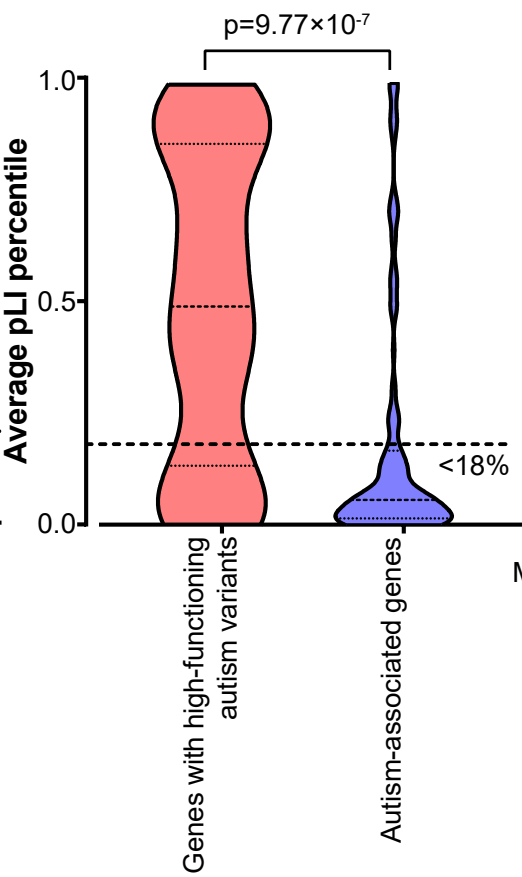

\section{Expression of autism genes in the developing brain}

Autism-associated genes

Genes with high-functioning autism variants Amygdala Cerebellum Cortex Hippocampus Striatum Thalamus Amygdala Cerebellum Cortex Hippocampus Striatum Thalamus

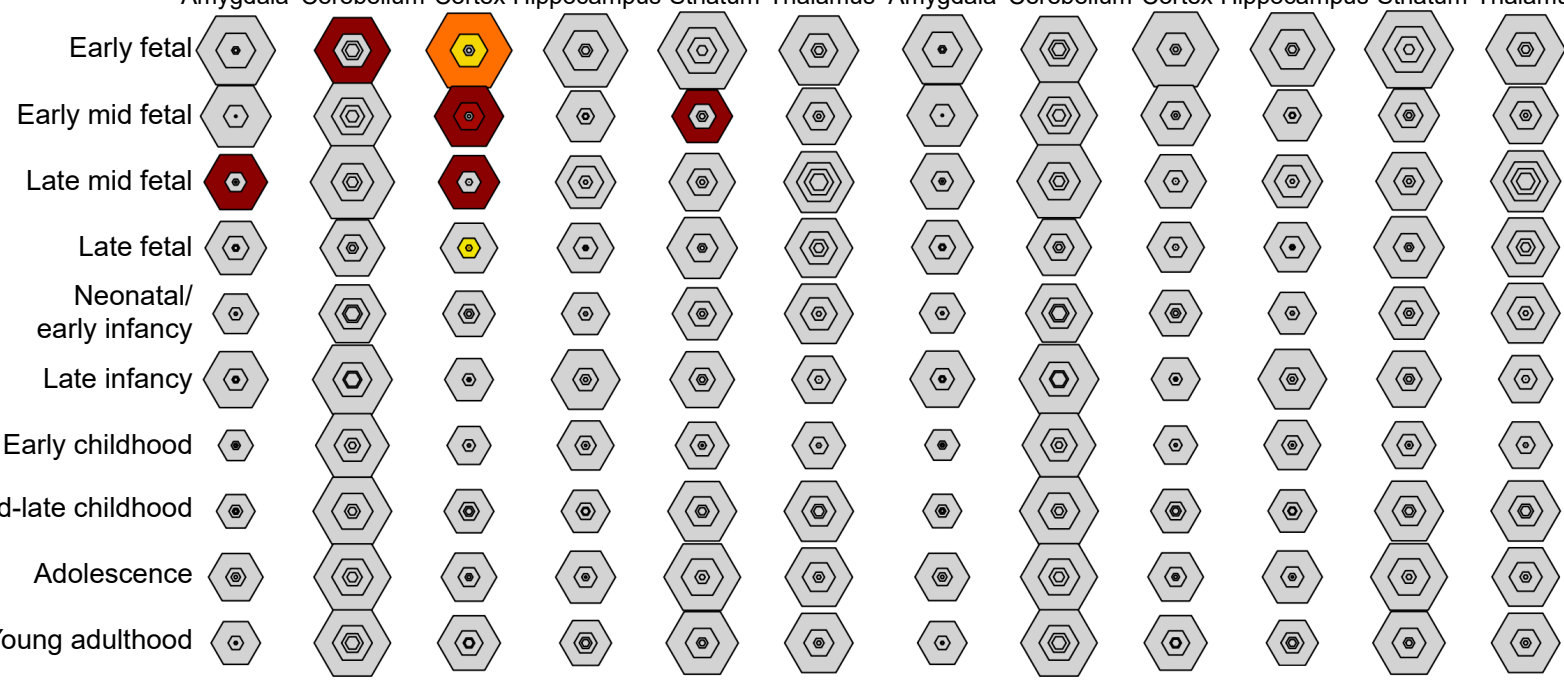

Enrichment (p-value) 0.1 0.075

Frequency of phenotypes in mouse models of autism genes

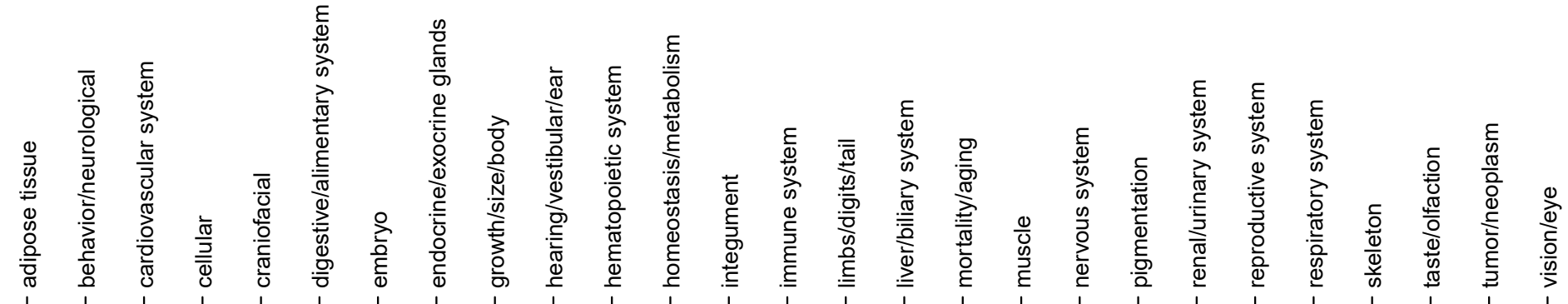

Frequency

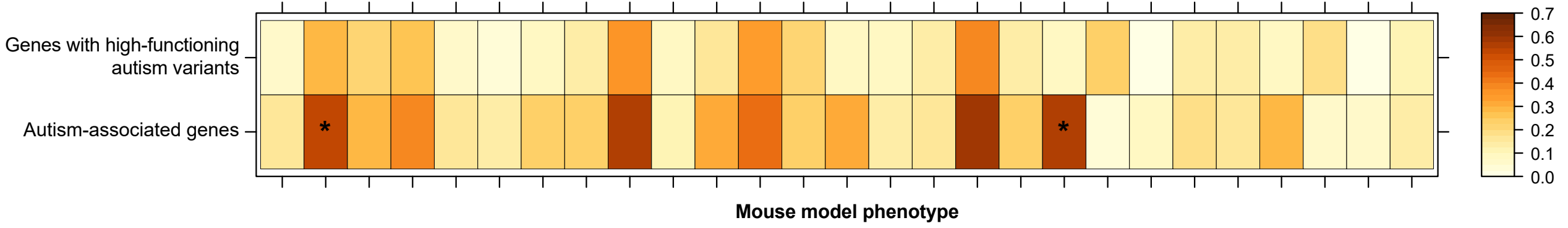

